\title{
Anatomical robotic prostatectomy: technical factors to achieve superb continence and erectile function
}

\author{
Zach S. Dovey, Ash K. Tewari \\ Department of Urology, Icahn School of Medicine at Mount Sinai, One Gustave L. Levy Place, New York, USA \\ Contributions: (I) Conception and design: ZS Dovey; (II) Administrative support: ZS Dovey; (III) Provision of study materials or patients: ZS Dovey; \\ (IV) Collection and assembly of data: ZS Dovey; (V) Data analysis and interpretation: ZS Dovey; (VI) Manuscript writing: All authors; (VII) Final \\ approval of manuscript: All authors. \\ Correspondence to: Professor Ash Tewari. Department of Urology, Icahn School of Medicine at Mount Sinai, One Gustave L. Levy Place, New York \\ 10029, USA. Email: ash.tewari@mountsinai.org.
}

\begin{abstract}
Debate continues as to the superiority of robotic versus open radical prostatectomy for the surgical treatment of localized prostate cancer. Despite this controversy, retrospective data from high volume centres has demonstrated RARP is associated with improved pentafecta outcomes with lower transfusion rates, less incontinence, lower positive surgical margins and improved potency. Advocates of robotic assisted radical prostatectomy (RARP) believe an enhanced visual field, the precision afforded by robotic technology as well as lack of bleeding, sharp dissection and delicate tissue handling lead to improved outcomes. Prostate Cancer is the second most common cancer diagnosed in men, and as the number of post-surgical patients increases, the complications of urinary incontinence and erectile dysfunction not only have a significant negative impact on patients' quality of life, but have become an expanding part of clinical practice. This article outlines what are believed to be the most important strategies based on anatomical knowledge and technical expertise, that allow robotic prostatectomists to achieve superb outcomes in urinary and erectile function.
\end{abstract}

Keywords: Prehabilitation; anatomy; technical expertise; adjunctive strategies; pentafecta outcomes

Submitted Sep 30, 2019. Accepted for publication Dec 30, 2019.

doi: 10.21037/tau.2020.01.15

View this article at: http://dx.doi.org/10.21037/tau.2020.01.15

\section{Introduction}

Although there remains some controversy as to the superiority of robotic assisted radical prostatectomy (RARP), retrospective data from high volume centres has demonstrated RARP is associated with improved pentafecta outcomes with lower transfusion rates, less incontinence, lower positive surgical margins and improved potency $(1,2)$. Advocates of RARP believe an enhanced visual field, the precision afforded by robotic technology as well as lack of bleeding, sharp dissection and delicate tissue handling explain these improved outcomes (1). Nevertheless, as the survivorship of prostate cancer post RARP increases, a significant number of patients still suffer with urinary and erectile dysfunction that in combination reduces their health related quality of life (HRQOL) (3). Improving continence and erectile function rates post RARP requires a detailed anatomical knowledge of the prostate and surrounding structures combined with technical expertise during the procedure. At our institution, a 3 Tesla (3T) multiparametric MRI of the prostate is mandatory before embarking on surgery. Pre-operative planning is crucial and is based on an individual's diagnostic parameters including histological grade, prostate tumor size, stage and location on MRI, assessed risk of extracapsular extension and seminal vesicle invasion (SVI), all of which we will discuss in more detail below. The oncological outcome is at the forefront of surgical planning, and within this framework, we plan detailed preservation and reconstruction of key anatomical landmarks including pelvic nerves, muscles 
of the bladder neck, pelvic floor and external sphincter, the detrusor apron and all the fibrous attachments of the prostate (4). In this article, we will discuss the anatomical landmarks and technical approaches for RARP separately, with specific reference to improving postoperative continence and potency. We also believe any discussion of improved postoperative outcomes for RARP is incomplete without outlining the importance of an integrative approach to patient care. In order to maximize the outcomes of a technically successful procedure, patients should be educated about the benefits of physical fitness and activity on post-operative HRQOL (3), using prehabilitation, and developing dietary, exercise and lifestyle habits that last long-term.

\section{Prehabilitation and rehabilitation}

Patients who are physically fit pre RARP, recover quicker and have better continence and HRQOL outcomes (5), and for other types of surgery have been shown to have reduced postoperative complications and all cause mortality. Treanor et al. [2018] conducted a metanalysis on prehabilitation versus usual care for cancer patients, including those for radical prostatectomy, using psychological support, education and various forms of exercise. They found Pelvic Floor Muscle Training (PFMT) and specifically Kegel exercises produced significant improvements in early continence at 3 months, but no significant change at 6 months (6). Pre surgical exercise was shown to reduce hospital stay and post-operative complications and psychological based prehabilitation improved wellbeing, immune function and mood in prostate cancer patients, as well as HRQOL across different cancers (7). Although these outcomes do not relate specifically to long-term improvements in continence and erectile function, they are linked to aetiological factors in their long-term decline (e.g., muscle tissue health, vascular disease, cardiovascular morbidity). On this basis, to maximize the technical success of an RARP procedure, we are strong advocates of prehabilitation including preoperative PFMT and Kegel exercises, and ask our patients pre RARP to have a consultation with an attending with expertise in Surgical Recovery for urinary and erectile function as standard of care.

\section{Achieving superb continence}

Reported rates of urinary incontinence (UI) vary significantly post RP, dependent on preoperative patient features, definitions of UI and methods of assessment, as well as the expertise of the surgeon and modality of RP $(7,8)$. If a 1 pad or less definition of continence is used, 89-92\% of men will be continent at 12 months (8) and up to $97 \%$ at 2 years (7). Defining continence as no pad usage, 69-96\% of patients achieve continence at 12 months in published series (8). Preoperative planning will highlight those patients at particular risk of a poorer outcome, such as older patients (9), those with higher BMIs and more comorbidities, those with lower urinary tract symptoms (LUTS) and those with higher prostate volumes (e.g., more than $70-80 \mathrm{cc})(8,9)$. The effect of a prior history of TURP on post-operative continence is less well described (8), and more recent reports have suggested that only age and prior LUTS were associated with poorer outcomes, but prostate size was not an independent predictor (10).

Having highlighted patients at risk, the surgeon can plan an approach to each specific anatomical landmark relevant to post-operative continence outcomes. For this article, we will consider both Anatomical Landmarks and Technical Strategies.

\section{Anatomical landmarks to achieve superb continence (see Table 1)}

The key anatomical landmarks can be divided into nerves (pelvic and pudendal), muscles (bladder neck and membranous urethra with inner lissosphincter and external rhabdosphincter, puboperinealis and levator ani) and periprostatic fibrous structures with attachments anteriorly (Retzius fibrous attachments and the detrusor apron), posteriorly (urethropelvic ligament and Denonvillier's fascia), laterally (periprostatic fascia, endopelvic fascia and arcus tendineus) and to the pubic bone $(4,11)$ (Table 1).

\section{Neuroanatomy}

The original work by Walsh and Donker [1982] (12) described the anatomy of nerves in relation to the prostate supplying the pelvic organs and penis in still born males. Since then, our understanding of the neuroanatomy of the pelvic plexus has made significant advances. The pelvic plexus receives input from sympathetic (T11L2) fibres controlling ejaculation as well as stimulating smooth muscle to increase the tone of the bladder neck and urethra and from parasympathetic (S2-4) fibres causing detrusor muscle contraction and erections. Cell bodies in the pelvic plexus give rise to post ganglionic fibres that are predominantly unmyelinated (13). The pelvic plexus is a bilateral rectangular plate shaped structure beginning 
Table 1 Anatomical structures involved in continence

\begin{tabular}{l} 
Nerves \\
\hline Pudendal nerves \\
Pelvic nerves \\
(a) Somatic nerves \\
(b) Autonomic inferior hypogastric neural plexus \\
Sympathetic nerves: T-11 to L-2 ganglia \\
Parasympathetic nerves: S-2 to S-4 spinal nerves \\
Muscles \\
Bladder neck and membranous urethra \\
(a) Inner lissosphincter \\
Longitudinal fibers \\
Circular fibers \\
(b) External rhabdosphincter \\
Puboperinealis \\
Levator ani \\
Fibrous structures \\
Anterior: Retzius fibrous attachments of the "Detrusor apron"
\end{tabular}

(a) Anterior musculotendinous with three layers

Anterior to the decussated pubococcygeal fibers

Middle layer to the dorsal vascular complex

Posterior layer to the dorsal vascular complex and prostate

(b) Puboprostatic ligament

Posterior

(a) Urethropelvic ligament

(b) Denonvilliers' fascia

Lateral

(a) Periprostatic fascia: multilayer

(b) Endopelvic fascia: Derived from pubococcygeus ligament

(c) Archus tendinosus

Pubic bone

Reproduced with kind permission of Sage publications; Arroyo et al., 2019 (4).

at the junction of distal pelvic ureter and vas deferens within the subperitoneal connective tissue, extending forward around the posterior and lateral parts of the seminal vesicle (14). Tewari et al. [2011] (15) detail three more surgically distinct zones of the pelvic plexus and it branches, which they describe as proximal neurovascular plate (PNP) associated with the seminal vesicle as above, the predominant neurovascular bundle (PNVB) located on the posterolateral angle between the prostate and rectum, and the accessory neural pathways (ANPs) within the lateral prostatic fascia. This "trizonal" neural hammock surrounds the prostate on each side (15). Somatic innervation to the pelvic floor musculature and external rhabdosphincter is from the pudendal nerve whose cell bodies are in "Onuf's nucleus" in the lateral border of the ventral horn of sacral spinal segments S2-4. Injury to the pelvic plexus or pudendal nerve branches by mechanisms of ischaemia, cautery, inflammation, or mechanical damage is potentially reversible, but injury to nerve cell bodies in the plexus itself is irreversible. With specific reference to continence, pelvic plexus injury may affect bladder neck and urethral function, and pudendal nerve branch injury may effect pelvic floor muscle and external sphincter function. The pudendal nerve branches may be compromised by suturing and dissection around the prostatic apex, and dorsal vein complex as well as during urethral transection by excessive traction on the prostate (16). A more detailed description of nerve sparing technique specifically is described below in the section of erectile function.

\section{Detrusor apron}

The "Detrusor Apron" was originally described by Santorini in 1724 (4). It extends distally from the anterior wall of the bladder over the surface of the prostate to the pubis, and towards the pelvic floor fuses laterally with the tendinous part of pubococcygeus and antero-medial aspect of the visceral endopelvic fascia, fixing both the prostate and bladder neck to the pubis. In this way, detrusor fibers extend past the bladder neck, spread around the prostate, and collect at the pubis (4). In a cadaveric study, examining the puboprostatic ligaments and prostatic apex in more detail, $\mathrm{Xu}$ et al. [2017] (17), described the detrusor apron in three layers. It has an anterior layer that fuses with pubococcygeal fibres, passing distally to fix to the pubic bone, a middle layer that passes distally in continuity with the fascia of the dorsal venous complex, and a posterior layer that forms the anterior fibromuscular layer of the prostate, passing forward to end by the external rhabdosphincter (18). None of these layers contain prostatic glandular tissue, and if indicated, can be safely dissected off the anterior aspect of the prostate to limit any injury to the sphincter complex and, by maintaining integrity of the tissues anterior to the urethra, improve continence post operatively (19). 


\section{Urethral sphincter}

The distal sphincteric unit consists of striated muscle of the external rhabdosphincter, smooth circular and longitudinal muscle of the inner lissosphincter, and associated paraurethral skeletal musculature with supporting fascia $(20,21)$. Careful apical dissection and suturing of the DVC will limit injury to this and surrounding structures, as well as branches of the distal pudendal nerve to associated musculature as described above.

\section{Membraneous urethra}

The membraneous urethra connects prostatic and bulbar urethra extending from the apex of the prostate piercing the urogenital diaphragm posterior to the pubic symphysis. The importance of membraneous urethral length (MUL) has been well described. In a recent metanalysis of four studies and over 1,700 patients, Mungovan et al. [2017] (22) found a clearly positive association between MUL and postoperative continence, and moreover, the longer the MUL by millimeter, the faster a patient's continence returned. Song et al. [2017] (23) studying a cohort of 190 men prospectively found preoperative MUL less than $16 \mathrm{~mm}$, post-operative MUL less than $14 \mathrm{~mm}$, and \% change greater than $18 \%$ were all associated with post-operative urinary incontinence at 6 months. Interestingly, Von Bodman et al., [2012] (20), in a retrospective analysis of 967 men, found not only MUL but also a close relationship of the membraneous urethra and levator muscle were important in gaining post-operative continence. Intuitively, this would suggest a "bulky", fit levator which has undergone preoperative Kegel exercises, would contribute to gaining continence post operatively. Moreover, it may also support the use of Kegel exercises long-term to maintain levator bulk and fitness for ongoing improvement in continence.

\section{Technical strategies to achieve superb continence}

\section{Bladder neck preservation}

The bladder neck has three layers of detrusor muscle (outer longitudinal, middle circular and inner longitudinal) with detrusor smooth muscle fibres of the trigone merging circumferentially with smooth muscle of the internal urethral sphincter. The bladder neck, trigone and internal urethral sphincter are jointly innervated by noradrenergic sympathetic fibres via the hypogastric nerve and pelvic plexus, increasing smooth muscle tone, and maintaining continence during the filling phase of micturition (11).
The technique for Bladder neck sparing (BNS) may be circumferential, anterior or lateral (24). Smolski et al. [2013] (24) in a systematic review of 33 studies including one prospective randomized blinded study, found BNS was associated with improved early and late continence as well as lower bladder neck stricture rates. Some studies have suggested BNS compromises oncological outcomes by increasing basal surgical margin rates (4) but a second systematic review with additional metanalysis confirmed the findings of Smolski et al. [2013] (24) and also found no difference in surgical margin rates or biochemical recurrence in BNS versus no BNS cohorts (11).

\section{Nerve preservation}

There are three major nerves of importance in the mechanism of continence; the pudendal nerve, the autonomic supply of the internal sphincter via hypogastric nerves and pelvic plexi, and the neurovascular bundle, which has been shown to provide some innervation for the membraneous urethra (25).

With regards to the pudendal nerve, Takenaka et al. [2005] (26) showed sphincteric branches of the pudendal nerve course in the distal endopelvic fascia close to the prostatic apex. This emphasizes the importance of meticulous apical dissection and careful over sewing of the DVC to avoid injury to these pudendal branches as well as preserving vascularity of the membranous urethra (26).

Reeves et al. [2015] (27) in a systematic review of over 13,700 men and 27 studies found NS was associated with better urinary continence rates up to 6 months postoperatively, with no difference between NS and NNS beyond 6 months. The benefit was most marked for bilateral NS compared with NNS. Interestingly, one of the studies showed NS improved UC in patients with preoperative ED (28), highlighting the importance of NS in all patients, not just for potency preservation. Srivastava et al. [2013] (29) found grades of NS described by Tewari et al. [2011] (15), correlated with early return of continence, so that conserving more nerves by dissecting closer to the prostatic capsule, correlated with earlier return of continence. Although this study was rebuked by Murphy and Costello [2013] (21), technically, it maybe that setting up the nerve sparing proximally by finding the appropriate tissue plane, can result in conservation of sympathetic fibres in the pelvic plexus that innervate the bladder neck and inner sphincter, so preserving this function more effectively. We will discuss nerve sparing technique in detail in the section on Erectile Function below. 


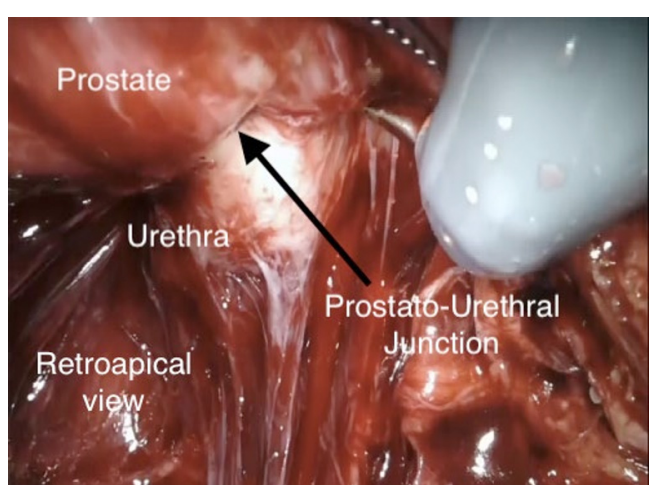

Figure 1 Retroapical view of the prostatourethral junction using 30 degree up lens. [modified and reproduced with kind permission of Sage Publications; Martini and Tewari, 2019 (36)].

\section{Apical dissection and retro-apical urethral transection}

Crucial steps in the apical dissection and dissection of structures associated with the prostatic apex and external sphincter include preservation of the endopelvic fascia and puboprostatic ligaments, athermal division of the DVC, selective suture ligature (SSL) and over sewing of the DVC, meticulous dissection of the apex itself in close proximity to the external sphincter, and preservation of maximal urethral length (11).

Takena et al. [2005] (26) demonstrated endopelvic fascia over levator ani muscle contains many smooth muscle fibers that mix with the external sphincter, as well small branches of the pudendal nerve to the external sphincter near the apex at 5 and 7 o'clock. With sparing of the endopelvic fascia, they demonstrated a UC rate of $83 \%, 96 \%$, and $100 \%$ at 3, 6, and 9 months respectively after RARP. These findings were supported by Van der Poel et al. [2009] (30) who found in 151 patients who underwent RARP, endopelvic fascial sparing was independently predictive of $\mathrm{UC}$ at 6 and 12 months.

Preservation of the puboprostatic ligaments, was shown to improve early incontinence by Stolzenberg et al. [2006] (31) with $\%$ of patients who had ligament preservation achieving continence at 3 months compared to $48 \%$ who didn't. The technique of puboprostatic preservation is closely aligned to proximal athermic division of the DVC with oversewing or SSL. Interestingly, SSL has been shown to improve 5 -month continence rates in 303 men who had this technical modification compared to 240 men who didn't (32), after adjusting for BMI, age, preoperative LUTS and NS or NNS approaches.

Hoshi et al. [2013] (33) also demonstrated improved UC rates with combined puboprostatic preservation, NS and SSL of the DVC.

Assessing combined meticulous apical dissection with NS in a retrospective study of nearly 18,500 men, Michl et al. [2016] (34) showed NS with careful apical dissection to avoid injury to the external sphincter improved both early and long-term UC rates.

We have discussed the benefits of MUL on postoperative continence above. An interesting prospective double cohort study by Borin et al. [2007] (35) showed in the first group of 200 cases, with transection of the urethra at the prostatourethral junction, the PSM was $17.6 \%$ (74\% apical) with continence at 6 months of $89 \%$. In the second group of 200 cases, with transection 3-6 mm distal to the prostatourethral junction, the PSM was 7.5\% (apical 5.5\%) with continence at 6 months of $91 \%$. They concluded it is possible to be more aggressive in urethral transection, reducing PSMs, without affecting continence. An alternative method of retro-apical dissection was demonstrated by Martini et al. [2019] (36) using a synchronous, posterior to anterior approach with a $30^{\circ}$ up lens. This allows improved visualization of prostatourethral junction, also maintaining preservation of the distal NVB, without affecting PSMs (Figure 1).

\section{Total anatomical reconstruction (Figure 2)}

Martini et al. [2019] (36) describe a technique of "Total Anatomical Reconstruction" undertaken by Dr. Tewari, in order to combine the benefits of both posterior and anterior reconstruction (28). Reconstruction of Denonvillier's fascia and the posterior bladder wall is the initial step in this technique. Reconstruction of the posterior plate first, fixes and provides support to the posterior urethra (36). In keeping with the Pagano principle, a muscular flap is created behind the bladder neck, and a midline stitch is placed on right and left detrusor flaps behind the bladder neck with an additional suture to a retrotrigonal flap for more support $(36,37)$. More suturing anchors the retrotrigonal layer and DF to the posterior urethra, in line with the Rocco principle (38) (Figure 2A), following which posterior wall of the bladder is closed (Figure $2 B$ ) and the vesicourethral anastomosis is performed tension free using a continuous suture (39). Total anatomical reconstruction is then concluded with a running suture from the new bladder neck to the arcus tendineus $(36,39)$ (Figure 2C,D). Using this technique, Tewari et al. [2008] (39) showed improved UC recovery post operatively, with anterior and posterior reconstruction combined, outperforming posterior 

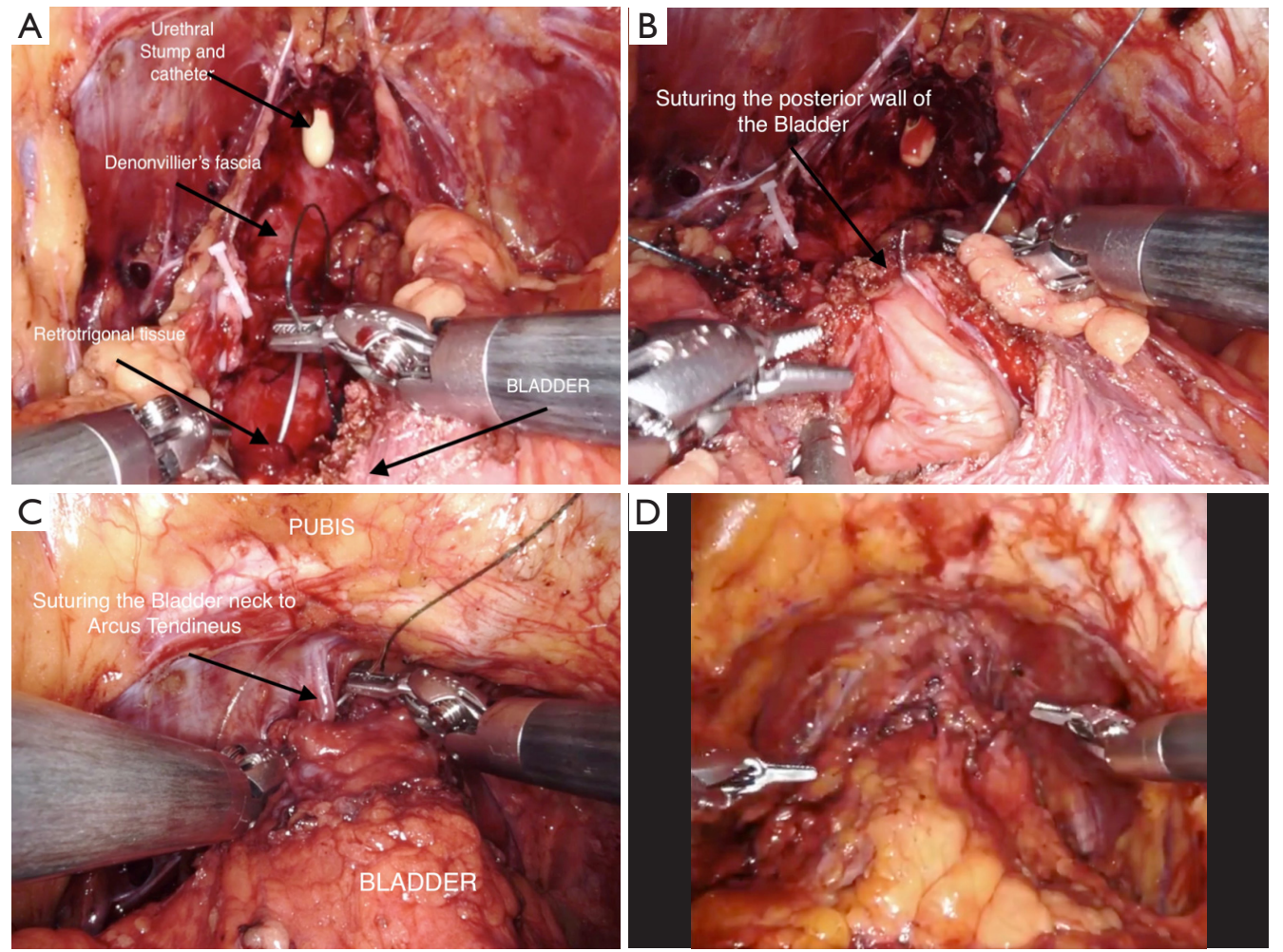

Figure 2 Total anatomical reconstruction. (A) posterior plate; Denonvillier's fascial layer; (B) posterior plate; bladder reconstruction; (C) anterior plate; (D) view of total anatomical reconstruction.

reconstruction alone.

These findings have been supported by two randomized control trials. Hurtes et al. [2012] (40) in 74 patients showed early UC rates at 1 and 3 months were better for those undergoing reconstruction compared to those who $\operatorname{did} \operatorname{not}(\mathrm{P}=0.047$ and $\mathrm{P}=0.016$, respectively). Student et al. [2017] (41), in their cohort of 66 patients randomized to TAR versus standard RALP, showed UC rates post-surgery were higher in the reconstructed group at 24 hours $(21.9 \%$ vs. $5.9 \% ; \mathrm{P}=0.079), 2$ weeks $(43.8 \%$ vs. $11.8 \% ; \mathrm{P}=0.005)$, 4 weeks $(62.5 \%$ vs. $14.7 \%$; $\mathrm{P}<0.001), 8$ weeks $(68.8 \%$ vs. $20.6 \% ; \mathrm{P}<0.01), 6$ months (75\% vs. $44.1 \% ; \mathrm{P}=0.013)$, and 12 months (86.66\% vs. $61.29 \% ; \mathrm{P}=0.04)$. Other prospective cohort studies have demonstrated similar findings $(25,42)$.

Summarising succinctly technical strategies used to achieve excellent continence results in a nonsystematic review of the literature from January 2000 to October 2018, Zattoni et al. [2019] emphasise the importance of a combination of all three approaches as appropriate allowing for preservation, reinforcement and reconstruction of all dissected anatomical structures in the pelvis (11).

\section{Achieving superb erectile function}

The aetiology of Erectile Dysfunction post RARP is direct injury to PNP, PNB or ANPs (15) or secondary injury resulting from traction, compression or cautery. Arteriogenic and venogenic factors with secondary ischaemia may play a role, and also contribute to subsequent chronic cavernosal tissue changes (43).

\section{Pre RARP assessment and technical planning}

As well as the preoperative preparation and prehabilitation described above, the use of a preoperative MRI based nomogram (43) predicting side specific extracapsular extension (ECE) and SVI is crucial. Based on the risk of ECE and SVI, an individual patients' side specific grade of nerve sparing can be planned (as well as helping to decide whether detrusor apron sparing should be undertaken) (15).

\section{Nerve sparing surgery}

The evolution of nerve sparing techniques has occurred 
Table 2 Grades of nerve sparing

Grade 1 NS: dissection is continued in a plane beneath the inconsistent loose layer of vascular fascia onto the prostate pseudocapsule itself, maximising sparing of the ANPs

Grade 2 NS: dissection is through the venous layer of fascia, beneath the formal LPF, which will conserve the majority of ANPs

Grade 3 NS: dissection is outside the LPF, sparing levator fascia, and conserving the PNB, but not ANPs

Grade 4 NS or non-NS: the LPF is excised and left on the prostate specimen

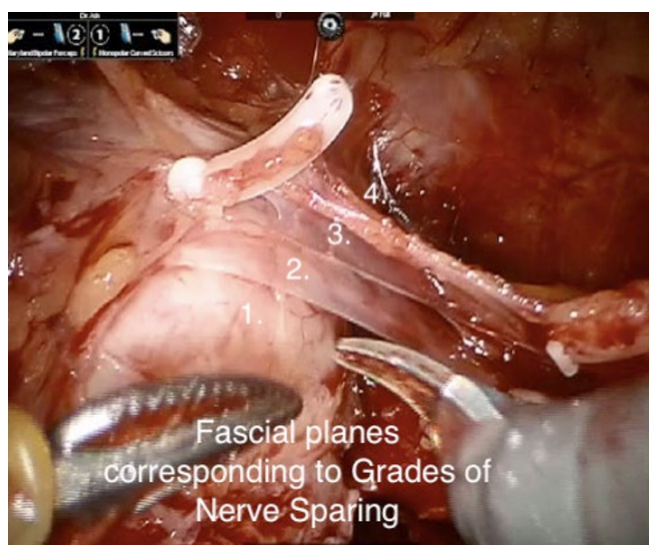

Figure 3 Nerve sparing fascial planes corresponding to grades of nerve sparing [modified and reproduced with kind permission of Sage Publications; Martini and Tewari, 2019 (36)].

in tandem with a more detailed understanding of the neuroanatomy. Tewari et al. [2011] (15) has described 4 grades of nerve sparing relating to fascial layers on the surface of the prostate, and the accessory nerve pathways (ANPs) they are proposed to contain. These ANPs form one part of the trizonal neuroanatomy of autonomic innervation for erectile function and the grade of nerve sparing is predetermined by an MRI based nomogram (43). Importantly, the NS technique begins at dissection of the SVs and vasa, and continues with dissection of Denonvillier's Fascia (DF) and distally towards the apex of the prostate (36). After dividing the posterior wall of the bladder neck, a retrotrigonal layer of fascia is incised onto the SVs and vasa deferentia. From this point on, we recommend athermic sharp dissection and clip application for bleeding vessels. This is specifically to avoid damage to the cell bodies and autonomic fibres of the pelvic plexus, especially at the tips and lateral aspect of the SVs where the pelvic plexus forms the PNP. Distally, nerve fibres continue in the PNVB and ANPs. Injury to the nerve fibres at any point may have potential for limited recovery with axonal regrowth after wallerian degeneration, but injury to the cell bodies in the PNP is irreversible (36).

Once the SVs have been isolated and elevated, DF is exposed inferior to the posterior surface of the prostate. The level of dissection proceeds dependent on risk of ECE and associated recommended Grade of NS, and DF may be entirely preserved, with a plane opened between DF and the posterior prostatic pseudocapsule, partly preserved with a dissection plane between anterior and posterior layers of DF (interfascial dissection), or not incised, leaving all layers of DF on the prostate (extrafascial dissection) (36). These planes are then developed laterally leading to 4 separate grades of NS $(15,36)$, determined by three layers of lateral prostatic fascia (LPF) (Table 2 and Figure 3). The three layers of the LPF have been described as, firstly, a loose layer of fascia containing arteries and veins, lying over the prostatic pseudocapsule: secondly, a clearly defined layer which is the lateral prostatic fascia itself, and thirdly, the outer layer which is levator fascia. Dissecting through these fascial planes not only sweeps the PNB laterally, but has also been proposed to spare the fibres of the ANPs. Based on these three fascial planes, the 4 grades of nerve sparing are; (Table 2 and Figure 3).

(I) Grade 1 NS: dissection is continued in a plane beneath the inconsistent loose layer of vascular fascia onto the prostate pseudocapsule itself, maximising sparing of the ANPs;

(II) Grade 2 NS: dissection is through the venous layer of fascia, beneath the formal LPF, which will conserve the majority of ANPs;

(III) Grade 3 NS: dissection is outside the LPF, sparing levator fascia, and conserving the PNB, but not ANPs;

(IV) Grade 4 NS or non-NS: all fascial layers are excised and left on the prostate specimen, including sacrificing the NVB.

The role of ANP sparing in improving post-operative erectile function remains somewhat controversial, and disparity has been demonstrated between the technical success of NS procedures and genuine improvements in outcomes. Nevertheless, they have been shown to conduct impulses to cavernosal tissue independent of the PNB (44), and it is possible there may be individual variability in their presence and function that may partially explain any disparity in outcomes (45). Moreover, conserving the ANPs distally has been shown to improve outcomes (38), and can be done reliably by performing a "high anterior release", dissecting over the prostate medially and anteriorly near the apex. 


\section{Arterial sparing surgery}

The origin of prostate arteries is the internal pudendal artery in $(35-56 \%)$, the gluteal-pudendal trunk $(15-28 \%)$, or the obturator artery (10-12\%) (46). The artery divides on both sides into a posterior branch, supplying the prostate base, the seminal vesicles, the vas deferens and an anterior branch supplying the prostate and prostatic apex. The anterior pedicle gives off small anterior capsular branches which contribute to penile blood supply, and so may be involved in post-operative ED if damaged (47). Accessory pudendal arteries (APAs) are present in 4-75\% of cases (48), arising from the external iliac, internal iliac or obturator arteries and usually course on the endopelvic fascia beneath the pubic arch to the anterolateral prostatic apex (49). APAs may be the only blood supply to the corpora cavernosa and sacrificing them can compromise penile blood supply, as well as being associated with post-surgery ED in nearly $60 \%$ of cases $(48,50)$. In a study by Box et al. [2010] (51) of 200 patients treated with RARP; 19 patients had APAs that were ligated, with $95 \%$ of them showing recovery of ED after surgery.

\section{Adjuncts to improve nerve sparing surgery}

There are a number of adjunctive techniques which have been used to improve nerve sparing, including:

NeuroSAFE frozen section. At our Institution we routinely send off the prostate (with seminal vesicles) for frozen section of each of the quadrants of the specimen margins in a technique known as "Neurovascular Structure-Adjacent Frozen Section Examination" (NeuroSAFE), originally described by Schlomm et al. [2012] (52). If the frozen section analysis reveals a positive surgical margin, more tissue can be removed at the appropriate anatomical location. In keeping with the original study, there is no delay in surgery as the posterior reconstruction, vesico-urethral anastomosis, anterior reconstruction and bilateral lymph node dissection can be performed while the report is being processed. We support the view that this technique affords the operating surgeon an aggressive approach to nerve sparing without ultimately compromising positive surgical margin rates.

Other techniques to improve nerve sparing. Seminal vesicle preservation has been suggested to limit injury to the PNP, but is not used in our institution and did not show any real benefit in either sexual function or continence in a randomized control trial in 140 men (53). Athermal technique is important once the vasa have been divided (50), to limit injury from electrocautery, and limiting countertraction on the neurovascular bundle and adjacent tissues is also of benefit (54). Hypothermic robotic radical prostatectomy has been used to improve outcomes with cold intracorporeal irrigation and endorectal colling to 4 degrees (55). This is to reduce tissue inflammation analogous to techniques in cardiac and neurosurgery, and was demonstrated to improve potency measured at 15 months (56). Some groups have shown promising early returns in potency and continence with dehydrated human Amnion-Chorion membrane (dHACM) wrapped around the neurovascular bundle intra-operatively to improve neural regeneration (56). The surgical insult on the neurovascular bundle causes not only a direct injury but also subsequent oedema, inflammation, acidosis, further hypoxia and secondary neural damage during the healing process. AHACM is rich in cytokines, growth factors and can also act as a physical barrier protecting the NVBs from this delayed injury. Another study using propensity analysis on 235 patients, also demonstrated improved early potency with $\mathrm{DHACM}$ wrapped around the NVBs intraoperatively (56), and a Clinical Trial is planned at our institution to further investigate its efficacy.

\section{Conclusions}

Radical prostatectomy continues to evolve as a procedure, with advances in our anatomical knowledge of the pelvic plexus, pelvic floor and penile innervation, and apical periprostatic tissues combined with the magnification, precision and dexterity afforded by robotic technology. We suggest to maximize the technical success of the procedure, prehabilitation should be standard of care alongside a detailed knowledge of the anatomy and surgical expertise. To further minimize post-operative incontinence and erectile dysfunction, novel adjunctive techniques may become useful in the future, such as the intraoperative application of amnion membrane. Taking a broader view, as we strive to improve all pentafecta outcomes, we believe radical prostatectomy will increasingly become a part of multimodal treatments, with neoadjuvant and adjuvant therapies, in keeping with the ultimate aim of reducing prostate cancer mortality.

\section{Acknowledgments}

Funding: None.

\section{Footnote}

Provenance and Peer Review: This article was commissioned by the Guest Editor (Ashok K. Hemal) for the series "Robotic-assisted Urologic Surgery" published in 
Translational Andrology and Urology. The article was sent for external peer review organized by the Guest Editor and the editorial office.

Conflicts of Interest: Both authors have completed the ICMJE uniform disclosure form (available at http://dx.doi. org/10.21037/tau.2020.01.15). The series "Robotic-assisted Urologic Surgery" was commissioned by the editorial office without any funding or sponsorship. AKT as of 2020 listed as company type, relationship type and financial. Uretheral Catheterless Radical Prostatectomy, Patent, No. DNA Based Bicistronic Vectors with Inducible and Constitutive Promoters - ID\#: 16060, Patent, No. High Intensity Focus Ultrasound and CPG-Brachyurys iRNA for Treatment of Prostate Cancer - ID\# 160403, Patent, No. Patent for a Catheterless Device and Approach, Patent, No. *Promaxo, Leadership position, Yes. *Promaxo, Equity Ownership, yes. Global Prostate Cancer Research Foundation, Leadership position, No. Kalyani Prostate Cancer Institute, Leadership Position, No. Prostate Cancer Foundation, Leadership Position, No. Roivant, Consultant, No. Blank Family Foundation, Grant, Yes. Intuitive Surgical, Scientific Study or Trial, Yes. Department of Defense (DOD), Scientific Study or Trial, Yes. AxoGen, Inc., Scientific Study or Trial, Yes. Oncovir, Inc - Poly ICLC, Scientific Study or Trial, Yes. National Institute of Health (NIH/DHHS), Scientific Study or Trial, Yes. National Cancer Institute, Scientific Study or Trial, Yes. National Institute on Drug Abuse, Scientific Study or Trial, Yes. Dr. Ash Tewari (the Principal Investigator in this study and Chairman of Milton and Carroll Petrie Department of Urology at the Icahn School of Medicine at Mount Sinai) owns equity in the form of stock certificates in Promaxo, for which he serves as an advisor. Promaxo is a privately traded company which develops MRI technology with a focus on prostate cancer. Kite Pharma, Scientific Study or Trial, Yes. Lumicell, Inc, Scientific Study or Trial, Yes. Dendreon, Scientific Study or Trial, Yes. *PROMAXO: Common Stock Certificate VALUE: 51,205\% SHARE RELATED PARTY: 0.63; Intuitive - no salary/see referenced COI's; Promaxo - no salary/investment - see referenced COI's; Kite Pharma: Serve as PI. Industry funded for research procedures. Site PI performance - no salary; Poly ICLC: serve as site PI. Phase I Study of IN SITU Autologous Vaccination Against Prostate Cancer With Intratumoral and Systemic HILTONOL (POLY-ICLC) Prior to Radical Prostatectomy. Product provided free of charge - no other funding. -- No salary. ZSD has no other conflicts of interest to declare.
Ethical Statement: The authors are accountable for all aspects of the work in ensuring that questions related to the accuracy or integrity of any part of the work are appropriately investigated and resolved.

Open Access Statement: This is an Open Access article distributed in accordance with the Creative Commons Attribution-NonCommercial-NoDerivs 4.0 International License (CC BY-NC-ND 4.0), which permits the noncommercial replication and distribution of the article with the strict proviso that no changes or edits are made and the original work is properly cited (including links to both the formal publication through the relevant DOI and the license). See: https://creativecommons.org/licenses/by-nc-nd/4.0/.

\section{References}

1. Coelho RF, Rocco B, Patel MB, et al. Retropubic, laparoscopic, and robot-assisted radical prostatectomy: a critical review of outcomes reported by high-volume centers. J Endourol 2010;24:2003-15.

2. Patel VR, Sivaraman A, Coelho RF, et al. Pentafecta: a new concept for reporting outcomes of robot-assisted laparoscopic radical prostatectomy. Eur Urol 2011;59:702-7.

3. Sanda MG, Dunn RL, Michalski J, et al. Quality of life and satisfaction with outcome among prostate-cancer survivors. N Engl J Med 2008;358:1250-61.

4. Arroyo C, Martini A, Wang J, et al. Anatomical, surgical and technical factors influencing continence after radical prostatectomy. Ther Adv Urol 2019;11:175628721881378.

5. Santa Mina D, Guglietti CL, Alibhai SMH, et al. The effect of meeting physical activity guidelines for cancer survivors on quality of life following radical prostatectomy for prostate cancer. J Cancer Surviv 2014;8:190-8.

6. Treanor C, Kyaw T, Donnelly M. An international review and meta-analysis of prehabilitation compared to usual care for cancer patients. J Cancer Surviv 2018;12:64-73.

7. Xu AJ, Taksler GB, Llukani E, et al. Long-term continence outcomes in men undergoing radical prostatectomy: a prospective 15 -year longitudinal study. J Urol 2018;200:626-32.

8. Ficarra V, Novara G, Rosen RC, et al. Systematic review and meta-analysis of studies reporting urinary continence recovery after robot-assisted radical prostatectomy. Eur Urol 2012;62:405-17.

9. Novara G, Ficarra V, D’Elia C, et al. Evaluating urinary continence and preoperative predictors of urinary continence after robot assisted laparoscopic radical 
prostatectomy. J Urol 2010;184:1028-33.

10. Lavigueur-Blouin H, Noriega AC, Valdivieso R, et al. Predictors of early continence following robot-assisted radical prostatectomy. Can Urol Assoc J 2015;9:e93-7.

11. Zattoni F, Artibani W, Patel V, et al. Technical innovations to optimize continence recovery after robotic assisted radical prostatectomy. Minerva Urol Nefrol 2019;71:324-38.

12. Walsh PC, Donker PJ. Impotence following radical prostatectomy: insight into etiology and prevention. J Urol 1982;128:492-7.

13. Jung J, Jo HW, Kwon H, et al. Clinical neuroanatomy and neurotransmitter-mediated regulation of penile erection. Int Neurourol J 2014;18:58-62.

14. Mauroy B, Demondion X, Drizenko A, et al. The inferior hypogastric plexus (pelvic plexus): its importance in neural preservation techniques. Surg Radiol Anat 2003;25:6-15.

15. Tewari AK, Srivastava A, Huang MW, et al. Anatomical grades of nerve sparing: a risk-stratified approach to neural-hammock sparing during robot-assisted radical prostatectomy (RARP). BJU Int 2011;108:984-92.

16. Narayan P, Konety B, Aslam K, et al. Neuroanatomy of the external urethral sphincter: implications for urinary continence preservation during radical prostate surgery. J Urol 1995;153:337-41.

17. Xu Z, Chapuis PH, Bokey L, et al. Nature and architecture of the puboprostatic ligament: a macro- and microscopic cadaveric study using epoxy sheet plastination. Urology 2017;110:263.e1-8.

18. Myers RP. Detrusor apron, associated vascular plexus, and avascular plane: relevance to radical retropubic prostatectomy - anatomic and surgical commentary. Urology 2002;59:472-9.

19. Takenaka A, Tewari AK, Leung RA, et al. Preservation of the puboprostatic collar and puboperineoplasty for early recovery of urinary continence after robotic prostatectomy: anatomic basis and preliminary outcomes. Eur Urol 2007;51:433-40.

20. Von Bodman C, Matsushita K, Savage C, et al. Recovery of urinary function after radical prostatectomy: predictors of urinary function on preoperative prostate magnetic resonance imaging. J Urol 2012;187:945-50.

21. Murphy DG, Costello AJ. How can the autonomic nervous system contribute to urinary continence following radical prostatectomy? A "boson-like" conundrum. Eur Urol 2013;63:445-7.

22. Mungovan SF, Sandhu JS, Akin O, et al. Preoperative Membranous Urethral Length Measurement and Continence Recovery Following Radical Prostatectomy:
A Systematic Review and Meta-analysis. Eur Urol 2017;71:368-78.

23. Song W, Kim CK, Park BK, et al. Impact of preoperative and postoperative membranous urethral length measured by 3 Tesla magnetic resonance imaging on urinary continence recovery after robotic-assisted radical prostatectomy. Can Urol Assoc J 2017;11:93.

24. Smolski M, Esler RC, Turo R, et al. Bladder neck sparing in radical prostatectomy. Indian J Urol 2013;29:338-44.

25. Sridhar AN, Abozaid M, Rajan P, et al. Surgical techniques to optimize early urinary continence recovery post robot assisted radical prostatectomy for prostate cancer. Curr Urol Rep 2017;18:71-8.

26. Takenaka A, Hara R, Soga H, et al. A novel technique for approaching the endopelvic fascia in retropubic radical prostatectomy, based on an anatomical study of fixed and fresh cadavers. BJU Int 2005;95:766-71.

27. Reeves F, Preece P, Kapoor J, et al. Preservation of the neurovascular bundles is associated with improved time to continence after radical prostatectomy but not long-term continence rates: results of a systematic review and metaanalysis. Eur Urol 2015;68:692-704.

28. Park YH, Kwon OS, Hong SH, et al. Effect of nervesparing radical prostatectomy on urinary continence in patients with preoperative erectile dysfunction. Int Neurourol J 2006;20:69-74.

29. Srivastava A, Chopra S, Pham A, et al. Effect of a riskstratified grade of nerve-sparing technique on early return of continence after robot-assisted laparoscopic radical prostatectomy. Eur Urol 2013;63:438-44.

30. van der Poel HG, de Blok W, Joshi N, et al. Preservation of lateral prostatic fascia is associated with urine continence after robotic-assisted prostatectomy. Eur Urol 2009;5 5:892-900.

31. Stolzenburg JU, Liatsikos EN, Rabenalt R, et al. Nerve sparing endoscopic extraperitoneal radical prostatectomy-effect of puboprostatic ligament preservation on early continence and positive margins. Eur Urol 2006;49:10311 ; discussion 111-2.

32. Lei Y, Alemozaffar M, Williams SB, et al. Athermal division and selective suture ligation of the dorsal vein complex during robot-assisted laparoscopic radical prostatectomy: description of technique and outcomes. Eur Urol 2011;59:235-43.

33. Hoshi A, Usui Y, Shimizu Y, et al. Dorsal vein complex preserving technique for intrafascial nervesparing laparoscopic radical prostatectomy. Int J Urol 2013;20:493-500.

34. Michl U, Tennstedt P, Feldmeier L, et al. Nerve- 
sparing surgery technique, not the preservation of the neurovascular bundles, leads to improved long-term continence rates after radical prostatectomy. Eur Urol 2016;69:584-9.

35. Borin JF, Skarecky DW, Narula N, et al. Impact of urethral stump length on continence and positive surgical margins in robot-assisted laparoscopic prostatectomy. Urology 2007;70:173-7.

36. Martini A, Tewari AK. Anatomic robotic prostatectomy: current best practice. Ther Adv Urol 2019;11:1756287218813789.

37. Tewari A, El-Hakim A, Rao S, et al. Identification of the retrotrigonal layer as a key anatomical landmark during robotically assisted radical prostatectomy. BJU Int 2006;98:829-32.

38. Rocco B, Gregori A, Stener S, et al. Posterior reconstruction of the rhabdosphincter allows a rapid recovery of continence after transperitoneal video laparoscopic radical prostatectomy. Eur Urol 2007;51:996-1003.

39. Tewari A, Jhaveri J, Rao S, et al. Total reconstruction of the vesico-urethral junction. BJU Int 2008;101:871-7.

40. Hurtes X, Rouprêt M, Vaessen C, et al. Anterior suspension combined with posterior reconstruction during robot-assisted laparoscopic prostatectomy improves early return of urinary continence: a prospective randomized multicentre trial. BJU Int 2012;110:875-83.

41. Student V, Vidlar A, Grepl M, et al. Advanced reconstruction of vesicourethral support (ARVUS) during robot-assisted radical prostatectomy: one-year functional outcomes in a two-group randomised controlled trial. Eur Urol 2017;71:822-30.

42. Porpiglia F, Bertolo R, Manfredi M, et al. Total anatomical reconstruction during robot assisted radical prostatectomy: implications on early recovery of urinary continence. Eur Urol 2016;69:485-95.

43. Martini A, Gupta A, Lewis SC, et al. Development and internal validation of a side specific, multiparametric magnetic resonance imaging-based nomogram for the prediction of extracapsular extension of prostate cancer. BJU Int 2018;122:1025-33.

44. Terada N, Arai Y, Kurokawa K, et al. Intraoperative electrical stimulation of cavernous nerves with monitoring of intracorporeal pressure to confirm nerve sparing during radical prostatectomy: early clinical results. Int J Urol 2003;10:251-6.

45. Kaul S, Savera A, Badani K, et al. Functional outcomes and oncological efficacy of Vattikuti Institute prostatectomy with Veil of Aphrodite nerve sparing: an analysis of 154 consecutive patients. BJU Int 2006;97:467-72.

46. Bilhim T, Pisco JM, Rio Tinto H, et al. Prostatic arterial supply: anatomic and imaging findings relevant for selective arterial embolization. J Vasc Interv Radiol 2012;23:1403-15.

47. Patel VR, Schatloff O, Chauhan S, et al. The role of the prostatic vasculature as a landmark for nerve sparing during robot-assisted radical prostatectomy. Eur Urol 2012;61:571-6.

48. Mulhall JP, Secin FP, Guillonneau B. Artery sparing radical prostatectomy--myth or reality? J Urol 2008;179:827-31.

49. Walz J, Burnett AL, Costello AJ, et al. A critical analysis of the current knowledge of surgical anatomy related to optimization of cancer control and preservation of continence and erection in candidates for radical prostatectomy. Eur Urol 2010;57:179-92.

50. Secin FP, Touijer K, Mulhall J, et al. Anatomy and preservation of accessory pudendal arteries in laparoscopic radical prostatectomy. Eur Urol 2007;51:1229-35.

51. Box GN, Kaplan AG, Rodriguez E Jr, et al. Sacrifice of accessory pudendal arteries in normally potent men during robot-assisted radical prostatectomy does not impact potency. J Sex Med 2010;7:298-303.

52. Schlomm T, Tennstedt P, Huxhold C, et al. Neurovascular structure-adjacent frozensection examination (NeuroSAFE) increases nerve-sparing frequency and reduces positive surgical margins in open and robot-assisted laparoscopic radical prostatectomy: Experience after 11,069 consecutive patients. Eur Urol 2012;62:333-40.

53. Gilbert SM, Dunn RL, Miller DC, et al. Functional outcomes following nerve sparing prostatectomy augmented with seminal vesicle sparing compared to standard nerve sparing prostatectomy: Results from a randomized controlled trial. J Urol 2017;198:600-7.

54. Kowalcyzk KJ, Huang AC, Hevelone ND, et al. Stepwise approach for nerve sparing without countertraction during robot-assisted radical prostatectomy: Technique and outcomes. Eur Urol 2011;60:536-47.

55. Finley DS, Chang A, Morales B, et al. Impact of regional hypothermia on urinary continence and potency after robot-assisted radical prostatectomy. J Endourol 2010;24:1111-6.

56. Ogaya-Pinies G, Palayapalam-Ganapathi H, Rogers T, et al. Can dehydrated human amnion/chorion membrane accelerate the return to potency after a nerve-sparing robotic-assisted radical prostatectomy? Propensity scorematched analysis. J Robot Surg 2018;12:235-43.

Cite this article as: Dovey ZS, Tewari AK. Anatomical robotic prostatectomy: technical factors to achieve superb continence and erectile function. Transl Androl Urol 2020;9(2):887-897. doi: $10.21037 /$ tau.2020.01.15 